\title{
ON PRE- AND POST-FRACTURE BEHAVIOUR OF LAMINATED GLASS UNDER BENDING
}

\author{
ALENA ZEMANOVÁ, JAROSLAV SCHMIDT, \& MICHAL ŠEJNOHA \\ Czech Technical University in Prague, Faculty of Civil Engineering, Department of Mechanics.
}

\begin{abstract}
The present study is focused on the application of phase-field modelling techniques to fracture simulation in laminated glass samples under bending. A damage model using a phase-field formulation of fracture is introduced and applied to three-layer laminated glass samples. The identification of material parameters of polymer foils and glass is also provided, based on a combined experimental and numerical analysis. Specifically, the results of small scale testing and the calibration of the constitutive models of polymer interlayers are discussed in connection to ethylen-vinyl acetate and polyvinyl butyral foils. The statistical data obtained by the evaluation of tensile strength of glass samples are used for the formulation of the tensile stress criterion. Therefore, a generalisation of the energetic formulation of phase-field models towards the stress-based criterion is employed here to simulate the fracture behaviour of laminated glass. The experimentally measured data are compared with the numerically derived response using the extreme values of tensile strength obtained. Then, the fracture response is analysed for one sample to support the proposed computational model and material parameters.

Keywords: Annealed glass, ethylen-vinyl acetate, heat-strengthened glass, laminated glass, phase-field damage model, polyvinyl butyral, rheometer, tensile strength in bending.
\end{abstract}

\section{INTRODUCTION}

In buildings, the transparent load-bearing structures typically appear in the form of laminates. Such types of multi-layer composites, consisting of glass commonly combined with polymer foils, open the way to increasing post-breakage resistance of structural units made of brittle glass. The critical aspect for the safety assessment of glass structures in various industries is to predict the fracture initiation and its propagation. Moreover, modelling of laminated glass is more demanding in comparison to the modelling of monolithic glass due to the time/temperature-dependent behaviour for polymer layers.

Many types of microstructure changes observed in materials can be described effectively by phase-field models. Over last years, the phase-field approach to brittle fracture has gained a lot of interest in computational mechanics. In this approach, discrete cracks are approximated by a crack phase field, and every discontinuity is smeared over some region. Hereby, crack initiation, propagation, and branching can be described with no need for crack tracking techniques. Therefore, this approach is employed here to predict the pre- and post-fracture behaviour of glass. In the present study, a combined experimental and numerical analysis of laminated glass samples is performed. Attention is limited to three-layer laminates made of two panes of annealed or heat- strengthened glass bonded by either ethylen-vinyl acetate (EVA) or polyvinyl butyral (PVB) interlayer foils.

To describe this research effort we organise the paper into the remaining four main sections corresponding to the experimental program and material properties identification in Section 2, numerical modelling in Section 3, validation in Section 4, and conclusions in Section 5. Prior to discussing the principal goal, we briefly summarise in Section 2.1 the calibration of the constitutive model of the two interlayers as they play an important role in a reliable prediction of the laminated glass response. Section 2.2 then describes the experimental program providing the data from bending tests on laminated glass samples. This experimental study is complemented with a numerical 
analysis described next, where the tensile stresses at failure are computed from known values of the critical load. Then, the whole data set is statistically evaluated and used in the phase-field model, which is introduced in Section 3.1. Its application to beam structures is briefly discussed in Section 3.2 and generalised for a stress-based criterion in Section 3.3. The validation is done for a laminated glass sample under four-point bending presented in Section 4.1 and its results are shown in Section 4.2 Finally, the conclusions for further research are summarised in Section 5.

\section{EXPERIMENTS AND MATERIAL PROPERTIES}

As mention in the introductory part, this section addresses two topics: the behaviour of two polymer foils, made of ethylen-vinyl acetate (EVA) and polyvinyl butyral (PVB), and the evaluation of tensile strength of glass. Based on these findings, the input parameters for the phase-field model will be set.

\subsection{Viscoelastic behaviour of polymer interlayers}

It has been shown that the time and temperature dependent viscoelastic response of these materials can be well described by the generalised Maxwell chain model plotted in Fig. 1(a), see e.g. [1]. This suggests the shear modulus at a given time instant $t$ be written in the form

$$
G(t)=G_{\infty}+\sum_{p=1}^{P} G_{p} e^{-G_{p} t / \eta_{p}}=f\left(G_{\infty},\left\{G_{p}\right\}_{p=1, \ldots ., P,}\left\{\eta_{p}\right\}_{p=1, \ldots . P}\right) .
$$

Assuming the relaxation times $\tau_{p}=\frac{\eta_{p}}{G_{p}}$ are set in advance leaves us with the shear moduli $G_{\infty}$ and $\left\{\mathrm{G}_{p}\right\}_{p=1, \ldots . . P}$ of the chain units to be calibrated. In the light of the adopted dynamic torsion experiments, it becomes useful to rewrite Eq. (1) in terms of the complex modulus $G^{*}$ as

$$
G^{*}(\omega, T)=G_{\infty}+\sum_{p=1}^{P} G_{p} \frac{a_{\mathrm{T}}(T) \omega^{2} \tau_{p}^{2}}{a_{\mathrm{T}}(T) \omega^{2} \tau_{p}^{2}+1}+\sum_{p=1}^{P} G_{p} \frac{a_{\mathrm{T}}(T) \omega \tau_{p}}{a_{\mathrm{T}}(T) \omega^{2} \tau_{p}^{2}+1},
$$

where $\omega$ is the harmonic frequency and $a_{\mathrm{T}}(T)$ is the temperature shift factor, transforming the experimental results obtained at a certain temperature $T$ to those corresponding to a chosen reference temperature $T_{\mathrm{R}}$. In this study, the parameter $a_{\mathrm{T}}(T)$ is evaluated from the Williams-Landel-Ferry (WLF) equation

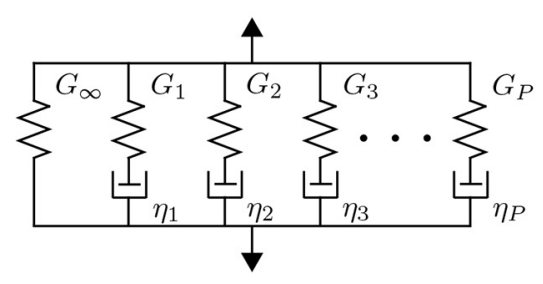

(a)

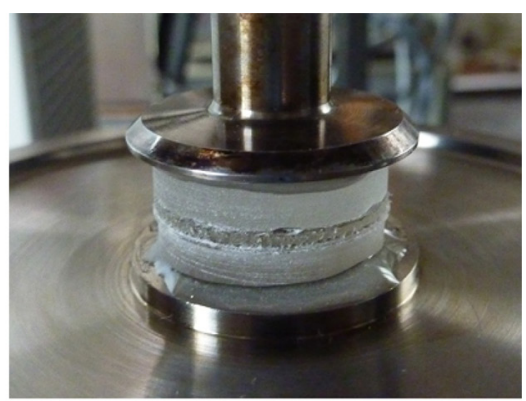

(b)

Figure 1: (a) Generalised Maxwell chain, (b) drilled out sample in rheometer. 


$$
\log a_{\mathrm{T}}(T)=\frac{-C_{1}\left(T-T_{\mathrm{R}}\right)}{C_{2}+T-T_{\mathrm{R}}}
$$

where $C_{1}, C_{2}$ are additional model parameters to be determined. The data entering the accompanying calibration procedure are provided in this study from the dynamic shear rheometer test as proposed, e.g. in [1].

Herein, the measurements were carried out using the HAAKE MARS apparatus. To that end, cylindrical samples drilled out from laminated glass plates were glued between two plates where the bottom plate is fixed whereas the top base is movable, see Fig. 1(b). The rheometer prescribes the torque of the top adapter around the longitudinal axis and records the resulting rotation. The built-in software then allows for calculating the complex shear modulus $G^{*}(\omega, T)$.

The measurements were performed on several samples for EVA and PVB foil sweeping the temperature domain of $10-60^{\circ} \mathrm{C}$ and the frequency domain of $0.001-100 \mathrm{~Hz}$. Unfortunately, the measured results at frequencies above $50 \mathrm{~Hz}$ were no longer physically acceptable and were therefore excluded from further analyses. The measured complex moduli were then compared with those provided by Eq. (2) together with Eq. (3) in the solution of a certain inverse problem. Details can be found in $[2,3]$. For illustration, the identified time-dependent shear moduli are plotted in Fig. 2 for both types of foils, PVB-based TROSIFOL ${ }^{\circledR}$ BG R20 and EVA-based EVALAM 80-120.

\subsection{Evaluation of tensile strength of glass}

Whilst the resistance of glass to compression is high, its resistance to tensile stresses is significantly lower because of surface flaws. This is also the cause of a relatively high scatter in the tensile strength measured experimentally on large structural specimens.

Our experimental study was performed on laminated glass samples with the nominal plane dimensions of $1,100 \times 360$ and the nominal thicknesses of glass/polymer/glass layers of $10 / 0.76 / 10 \mathrm{~mm}$. Four types of laminated glass samples were tested combining annealed glass (ANG) or heat-strengthen glass (HSG) plies with EVA and PVB foils. Both outer plies were made from the same type of glass. Two different scenarios were used, Fig. 3. Deflection-controlled four-point bending tests were performed on fourteen samples at the Faculty of Civil Engineering, Czech Technical University in Prague. In addition, load-controlled bending tests were carried out on 12 panes loaded under uniformly distributed pressure in vacuum chamber

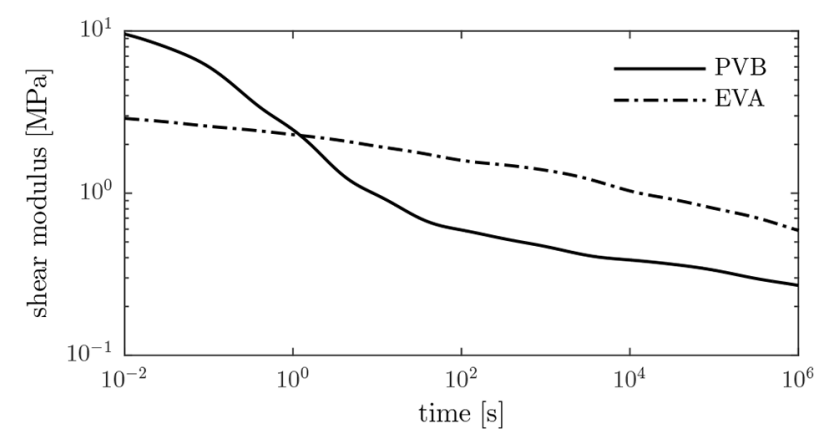

Figure 2: Relaxation modulus of PVB-based and EVA-based foil at $25^{\circ} \mathrm{C}$. 


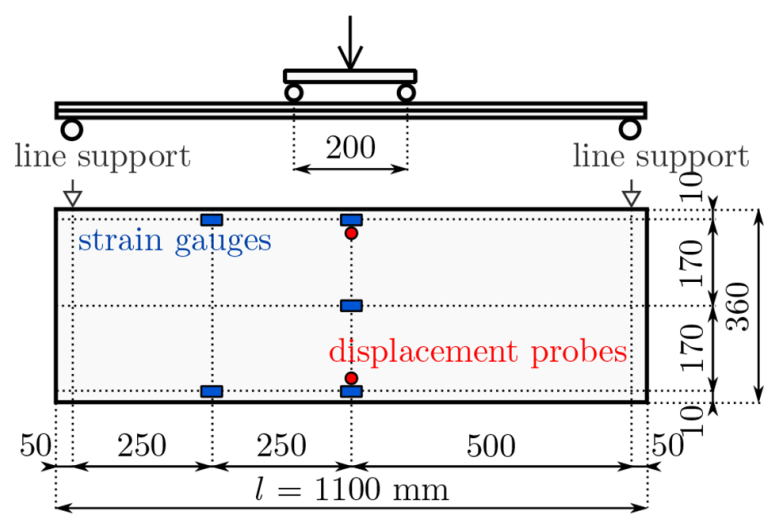

(a) Four-point bending test

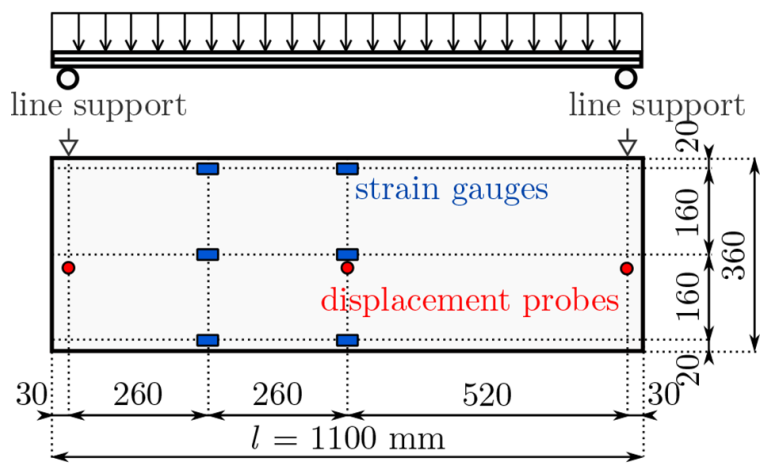

(b) Uniform bending test

Figure 3: Schema of bending tests.

at the AdMaS science centre, Brno University of Technology. The measured strains were converted into stresses using the Young modulus of glass, which was obtained from indentation tests performed at the Centre of Excellence Telč. As this quantity is not affected by the thermal tempering process, the measured value of $76.6 \mathrm{GPa}$ was used for both types of glass.

The experimental setup of the four-point bending test is illustrated in Fig. 3(a). Eight strain gauges LY 11-10/120 were attached to both glass plies, five on the upper surface in compression and three across the lower surface in tension at the midspan. Laminated glass samples were placed into MTS loading device and central deflections were measured by two displacement sensors. Rubber pads were placed between the supports and loading steel cylinders. The specimens were loaded in the displacement-controlled mode assuming the loading rate of 1.8 $\mathrm{mm} / \mathrm{min}$. The ambient temperature during the experimental testing was $25^{\circ} \mathrm{C}$. The tensile strengths in bending were considered equal to the failure stresses set from the extreme measured tensile strains at failure of the bottom glass ply. The strengths determined in such a way are within the range of 31-75 MPa for the annealed glass (10 specimens) and within the range of 92-105 MPa for the heat-strengthened glass (4 specimens).

The crack patterns of three samples are shown in Fig. 4 for illustration. Comparing the two patterns and strengths for the samples with annealed glass plies, it is obvious that the cracked 


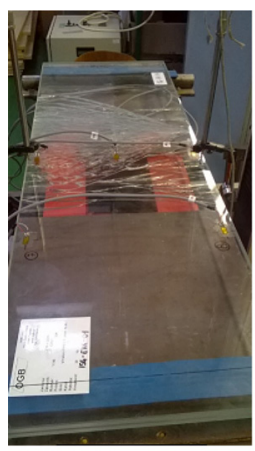

ANG $61 \mathrm{MPa}$

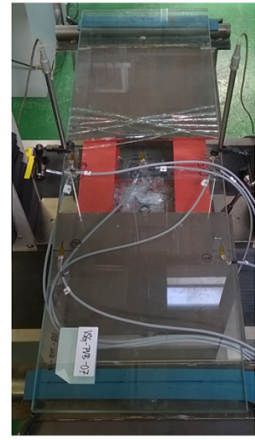

ANG $31 \mathrm{MPa}$

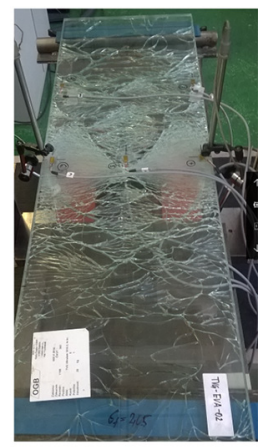

HSG $101 \mathrm{MPa}$

Figure 4: Crack patterns of three samples; courtesy of Tomáš Hána from CTU.

area is more localised for the sample with lower strength, which could be attributed to a local defect or to an asymmetry in the applied load. For the heat-strengthened glass, the cracks branch and spread across the whole area.

The second complementary experiments correspond to quasi-static bending tests in a vacuum chamber performed following the procedure described in [4]. The samples were placed into the chamber on two linear supports with soft pads made of rubber and loaded by the prescribed uniformed suction at $25^{\circ} \mathrm{C}$, Fig. $3(\mathrm{~b})$, resulting in tension in the bottom ply and compression in the top ply. For all samples, three strain gauges were placed on the upper surface at the midspan. In four cases, three additional strain gauges were placed at the quarter of the span and one at the centre of the bottom ply. The loading rate of $0.2-2 \mathrm{kPa} / \mathrm{s}$ was assumed. Because the loading was force-controlled, both glass plies broke at once. As this set of experiments was not initially proposed for the evaluation of tensile stresses, the maximum tensile strains were not measured for all samples. The missing values were replaced by the largest values of compressive strains at the midspan, which were always measured. Examining the strain distribution at the cross-section, and because the nominal thicknesses of the two glass plies are the same, allows us to assume that the tensile strains would have the same magnitudes as those in compression on the upper surface of the panel. This is supported by the fact that the deviations of the measured tensile and corresponding compressive strains were less than $5 \%$ for all the four-point bending tests and also for the four samples under uniform pressure with all seven strain gauges. The determined tensile strengths were within the range of 35-85 $\mathrm{MPa}$ for the annealed glass (6 specimens) and within the range of 115-132 MPa for the heat-strengthened glass (6 specimens).

Further, except the determination of strengths of glass from measured strains, the tensile stresses at failure were also computed from known values of the critical load. Such numerical or analytical calculations are, besides the strain measurements with strain gauges, one of the approaches used for the estimation of tensile stresses on monolithic glass plates [5]. However, this analysis is not so common for laminated glass samples because the unknown mechanical properties of interlayers make the determination of stress distribution in glass plies often impossible.

The details about the finite element solver developed for laminated glass and used for this numerical pre-fracture analysis can be found in [6] together with its verification. In short, the finite element model is based on a layer-wise formulation for beams including a viscoelastic constitutive law for the interalyer. Fourteen samples, corresponding to the scheme from Fig. 3(a), 
were analysed and the predicted failure stresses were within the range of 29-73 MPa for the annealed glass and 90-96 MPa for the heat-strengthened glass. The largest tensile stresses, corresponding to 12 tested samples under the experimental setup from Fig. 3(b), were within the range of 32-78 MPa for the annealed glass and 120-140 MPa for the heat-strengthened glass. The failure stress of one sample was not determined as the record of the magnitude of the loading pressure was defective. These numerical predictions match very well the values from experimental measurements. The errors between the experimental data and the numerical predictions are below $12 \%$, which is a very good agreement considering the fact that the discrepancy of the strains measured at midspan at the centre and near the edges is about $5 \%$, in a few cases up to $10 \%$.

The values of the tensile strengths for all samples independently can be found in [7]. Because the strength of glass strongly depends on many aspects, e.g. the condition of surface, the size of the glass element, the loading history, the residual stresses, or the environmental conditions, the test results are statistically analysed and evaluated; generally by fitting to a two-parameter Weibull distribution [8].

We combine the results of the two approaches mentioned above to obtain a larger data set for statistical evaluation of the tensile strength as both of them can be influenced by various uncertainties. Remember that 32 samples (16 measured, 16 calculated) for the annealed glass and 19 specimens (10 measured, 9 calculated) for the heat-strengthened glass were available.

The extreme values, the average, and the standard deviation of the data sets are summarised in Table 1 for both glass types. Compared with the standard values [9], the minimum of the tensile strengths for the annealed glass is under the characteristic value of $45 \mathrm{MPa}$ given by the draft Eurocode, whereas the smallest determined value of the strength of the heat-strengthened glass is about 30\% higher than the value of $70 \mathrm{MPa}$ suggested by the standard.

The occurrence of a given value of tensile strength is shown for the annealed and heat-strengthened glass in Fig. 5. For the heat-strengthened glass, it can be seen that the ranges of tensile stresses obtained by the four-point or uniform bending tests differ. The strengths of samples under uniform pressure are higher by about 20-30 MPa which could be caused by a different level of residual stresses developed during the tempering process. The histogram plots were complemented with two-parameter Weibull fits. The ability of these fits to characterize the data sets is illustrated by the Weibull probability plot. The tensile strength associated with the cumulative probability of 5\% corresponds to $28 \mathrm{MPa}$ for the annealed glass and $72 \mathrm{MPa}$ for the heat-strengthened glass, respectively. The mean values of the Weibull fits rounded to $1 \mathrm{MPa}$ match the average values in Table 1 . However, we should still bear in mind a relatively small data sets adopted in this study, which may also be the source of the observed, relatively large, scatter.

Table 1: Tensile strength of samples made of annealed or heat-strengthened glass.

\begin{tabular}{lcc}
\hline Value & Strength of ANG [MPa] & Strength of HSG [MPa] \\
\hline Average & 54 & 114 \\
Minimum & 29 & 90 \\
Maximum & 85 & 140 \\
Standard deviation & 15 & 17 \\
\hline
\end{tabular}



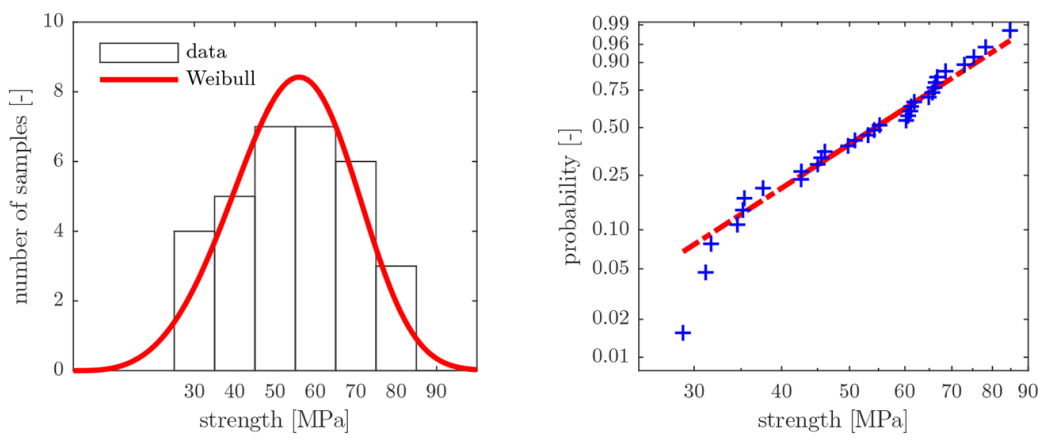

(a) Annealed glass
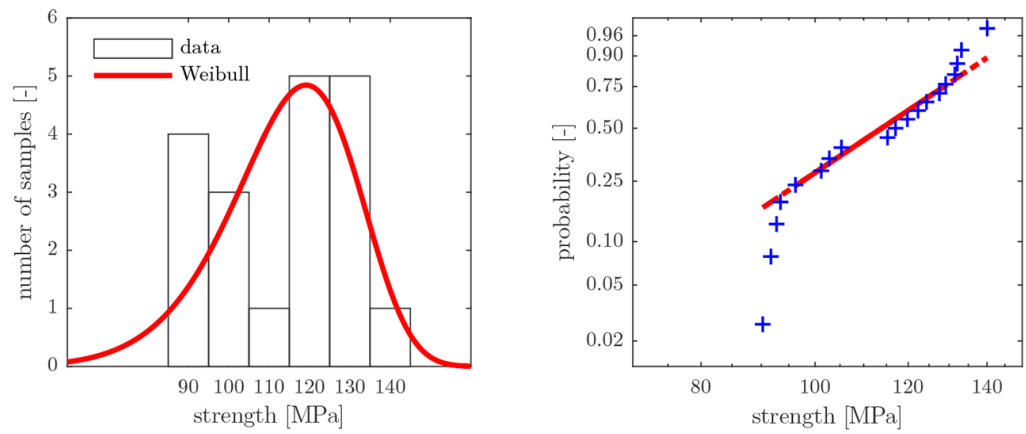

(b) Heat-strengthened glass

Figure 5: Weibull fit of tensile strengths (left) and corresponding probability (right).

\section{NUMERICAL ANALYSIS}

Having the material parameters of glass and interlayers, we will introduce the numerical model for laminated glass. Emphasis will be given to extension of the layer-wise finite element model from [6] towards fracture simulations. In this section, a brief introduction into a phase-field modelling of fracture is presented. An interested reader is referred for more details to, e.g. [10], [11], [12], [13].

\subsection{Variational formulation of fracture using phase-field model}

An elasticity problem can be formulated in the variational form by introducing the potential energy of the system $\mathcal{E}$. Assuming isotropic linear elasticity, the strain energy depends on the displacement gradient, $\nabla_{\text {sym }} \mathbf{u}$, which defines the small strain tensor $\varepsilon=\nabla_{\text {sym }} \mathbf{u}$, and the potential energy associated to the applied forces depends on the displacement vector $\mathbf{u}$. Then, the response of the system is represented by the displacement field $\mathbf{u}$ minimising the energy functional

$$
\mathbf{u}=\underset{\mathbf{u}}{\operatorname{argmin}}\left\{\mathcal{E}\left(\nabla_{\mathrm{sym}} \mathbf{u}, \mathbf{u}\right)\right\} .
$$

Similarly, the response of a fracturing system can be described by the displacement field $\mathbf{u}$ complemented with an internal discontinuity boundary $\Gamma \subset \Omega$, i.e. the crack surfaces in the domain $\Omega$ of the system. In this case, the process of crack initiation and propagation is governed by the minimisation problem of the energy functional, e.g. [10], 


$$
(\mathbf{u}, \Gamma)=\underset{(\mathbf{u}, \Gamma)}{\operatorname{argmin}}\left\{\mathcal{E}\left(\nabla_{\mathrm{sym}} \mathbf{u}, \mathbf{u}, \Gamma\right)\right\} .
$$

If we omit the energy associated with the applied forces, then the energy functional consists of two parts,

$$
\mathcal{E}(\varepsilon, \Gamma)=\int_{\Omega \backslash \Gamma} \psi_{\mathrm{e}}(\varepsilon) d \Omega+G_{\mathrm{c}} \int_{\Gamma} d \Gamma,
$$

the elastic strain energy computed from the elastic energy density $\psi_{\mathrm{e}}$ and the dissipated energy using the Griffith-type material fracture toughness $G_{\mathrm{c}}$.

In contrast to the discrete description of fracture, the phase-field models approximate the fracture energy by replacing the sharp discontinuity with a diffused crack model introduced by a phase field. The difference between a sharp and a diffused crack topology is shown in Fig. 6. An auxiliary variable $s$ called phase-field characterises the state of the material $(s=1$ corresponds to the intact material and $s=0$ to the fully cracked material). For a diffused crack, Fig. 6(b), the length scale parameter $l_{\mathrm{c}}$ controls the width of the diffuse interface, i.e. of the continuous approximation to the discrete crack. Then, the dissipated energy can be approximated by the volume integral

$$
G_{\mathrm{c}} \int_{\Gamma} d \Gamma \approx \int_{\Omega} \psi_{\mathrm{s}}(s, \nabla s) d \Omega=G_{\mathrm{c}} \int_{\Omega}\left(\frac{1}{4 l_{\mathrm{c}}}(1-s)^{2}+l_{\mathrm{c}}|\nabla s|^{2}\right) d \Omega .
$$

The crack surface density function $\psi_{\mathrm{s}}$ depends on the phase field $s$ and length scale parameter $l_{\mathrm{c}}$.

To achieve a state where the material cracks under tension and not under compression, the strain tensor $\varepsilon$ is decomposed in tensile $\varepsilon^{+}$and compressive $\varepsilon^{-}$contributions, e.g. by a spectral decomposition, [14]. Subsequently, the strain energy split holds

$$
\psi_{\mathrm{e}}(\varepsilon, s)=g(s) \psi_{\mathrm{e}}^{+}\left(\varepsilon^{+}\right)+\psi_{\mathrm{e}}^{-}\left(\varepsilon^{-}\right)
$$

where the tensile part $\psi^{+}\left(\varepsilon^{+}\right)$is modified by a degradation function $g(s)$ whereas the compressive part $\left.\psi_{\mathrm{e}}^{-}{ }^{-} \varepsilon^{-}\right)$stays unchanged.

Then, the regularised energy functional to be minimised reads

$$
\mathcal{E}(\varepsilon, s)=\int_{\Omega} g(s) \psi_{\mathrm{e}}^{+}\left(\varepsilon^{+}\right)+\psi_{\mathrm{e}}^{-}\left(\varepsilon^{-}\right)+G_{\mathrm{c}}\left(\frac{1}{4 l_{\mathrm{c}}}(1-s)^{2}+l_{\mathrm{c}}|\nabla s|^{2}\right) d \Omega,
$$

and the strong form of the corresponding governing equations is provided by

$$
\begin{gathered}
\operatorname{div} \boldsymbol{\sigma}=\mathbf{0} \\
\left(\frac{\mathcal{H}}{\frac{G_{c}}{4 l_{c}}}+1\right) s-4 l_{\mathrm{c}}^{2} \Delta s=1 .
\end{gathered}
$$

The first condition represents equilibrium equations for stresses $\sigma=\partial \psi_{\mathrm{e}} / \partial \varepsilon$, the second governs the evolution of the phase-field variable $s$. To ensure the irreversibility of the crack evolution, a history variable

$$
\mathcal{H}(t)=\max _{\tau \in[0, t]} \psi_{\mathrm{e}}^{+}(\tau)
$$




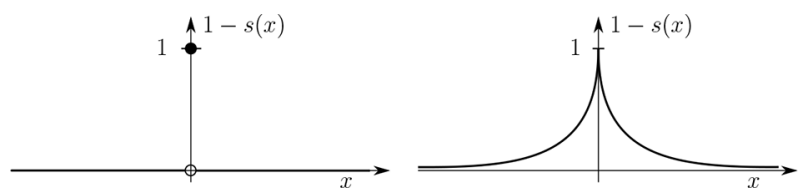

Figure 6: Crack topology: sharp crack (left), diffused crack (right).

is introduced, corresponding to the maximum tensile part of the elastic strain energy density for the time interval $[0, t]$. The solution of this coupled problem complemented with boundary conditions is found using a staggered approach, i.e. the equilibrium equation, Eq. (10), is solved first and then the phase-field equation, Eq. (11), for each time (or pseudo-time) instant [13].

\subsection{Phase-field formulation for Mindlin beams}

To reduce computational costs of the task, we use the refined beam elements to analyse the response of a multi-layer laminated glass structure, e.g. [6, 15, 16]. We follow the approach presented in [13] to obtain the tension-compression split based on the midsurface kinematic variables.

Using a degradation function $g(s)=s^{2}$, the decomposition of the strain energy functional beams can be written in the form

$$
\int_{\Omega} \psi_{\mathrm{e}}(\varepsilon, s) d \Omega=\underbrace{\int_{\Omega t} s^{2} E \varepsilon_{x}^{2}(x, z) d \Omega}_{\text {tension }}+\underbrace{\int_{\Omega c} E \varepsilon_{x}^{2}(x, z) d \Omega}_{\text {compression }}+\underbrace{\int_{\Omega^{*}} s^{2} G \gamma_{x z}^{2}(x) d \Omega,}_{\text {shear }}
$$

where $\varepsilon_{x}$ and $\gamma_{x z}$ are the non-zero components of the strain tensor and parameters $E$ and $G$ stand for the Young and shear moduli of an elastic material, respectively. The volume $\Omega_{*}$ corresponds to the effective shear area $A_{*}, \Omega_{t}$ to the cross-sectional area in tension $A_{t}$, and $\Omega_{c}$ to the area in compression $A_{c}$, Fig. 7. It can be seen that to decompose the strain energy functional means to find the position of the neutral axis $z_{N}$.

\subsection{Phase-field formulation based on tensile stress criterion}

To easily incorporate the results of the tensile strength evaluation from Section 2.2 into our numerical model based on the phase-field formulation for brittle fracture, the governing equations are adjusted using the tensile stress criterion according to [12].
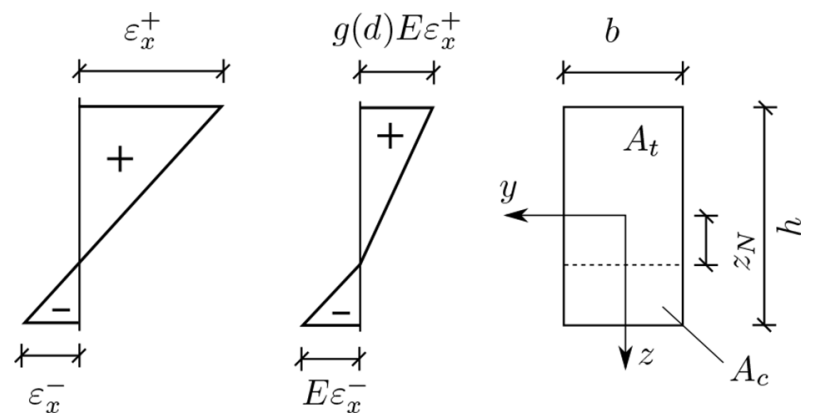

Figure 7: Scheme of the tensile-compression split. 
Therefore, the energetic formulation describing the phase-field evolution by Eq. (11) is replaced with

$$
\left(\left\langle\frac{\mathcal{H}}{\psi_{\mathrm{c}}}-1\right\rangle+1\right) s-4 l_{\mathrm{c}}^{2} \Delta s=1,
$$

where the Macaulay brackets define a ramp function as follows $\langle a\rangle=(a+|a|) / 2$. Finally, the critical fracture energy per unit volume $\psi_{\mathrm{c}}$ is related to a critical fracture stress $\sigma_{\mathrm{c}}$ by the expression

$$
\psi_{\mathrm{c}}=\frac{\sigma_{\mathrm{c}}^{2}}{2 E} .
$$

In this study, the extreme or actual tensile strengths from Section 2.2 are used as this critical stress. The advantage of this formulation is that it is simple and no damage appears in the elastic range. On the contrary, the formulation stops to be variationally consistent.

\section{VALIDATION AND DISCUSSION}

In this section, the experimentally measured data are compared with those derived numerically to support the proposed computational model.

\subsection{Example}

The validation was done for one four-point bending test, Fig. 3(a). The laminated glass sample was composed by two ANG layers connected with an EVA interlayer. The thicknesses of glass layers were reduced compared to the nominal values to obtain the measured overall thickness of the laminated sample, i.e. the value of $9.8 \mathrm{~mm}$ was used for a glass layer.

The parameters used in the simulation are summarised in Table 2. As the duration of loading was small, the viscoelastic effects were neglected in this example and the interlayer material is approximately replaced by an equivalent elastic solid [17]. Thus, the shear modulus of the interlayer was evaluated in the middle of each time interval independently according to Eq. (1) from the Prony series for EVA, Fig. 2.

Three critical stresses were tested: the actual tensile strength measured for the given sample $(61 \mathrm{MPa})$ and further, the two extreme values of the strength data set for annealed glass, Table 1.

\subsection{Results}

The response of the sample under four-point bending can by illustrated by a deflection/force diagram. In Fig. 8(left), the experimentally measured deflections (Exp 1 and Exp 2)

Table 2: Input data.

\begin{tabular}{llll}
\hline Parameter & Value & Parameter & Value \\
\hline Young's mod. of glass $E_{1}$ & $76.6 \mathrm{GPa}$ & Length scale p. $l_{\mathrm{c}}$ & $0.01 \mathrm{~m}$ \\
Poisson's ratio of glass $v_{1}$ & 0.22 & Elements per length $n$ & 100 \\
Shear mod. of EVA $G_{2}$ & from Eq. (1) & Critical stresses $\sigma_{\mathrm{c}}$ & $29 / 61 / 85 \mathrm{MPa}$ \\
Poisson's ratio of EVA $v_{2}$ & 0.49 & Number of time steps & 50 \\
\hline
\end{tabular}



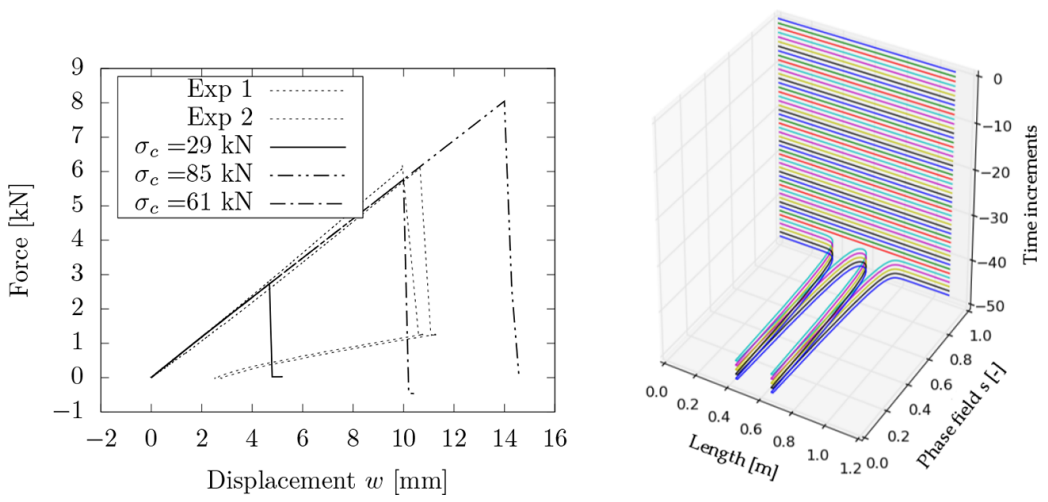

Figure 8: Deflection/force diagram (left) and evolution of phase field (right)

correspond to the data records of two displacement sensors at the midspan of the beam. After the fracture of one glass layer, the sample was unloaded, which is visible in the graph.

It is obvious that the range of the numerical response computed for the extreme values of the critical stresses listed above is substantial. The largest central deflection at the moment of fracture can be almost a triple of the value corresponding to the minimum tensile strength. A good agreement with the experimental data was found for the actual tensile strength. In the pre-critical range, the model response corresponds to the experimental measurement very well.

The phase-field evolution for the individual time steps is plotted across the length of the bottom layer of the beam in Fig. 8 (right). In the post-critical stage, the numerical model predicts faster descent, which could be given by boundary conditions of the task. As the laminated glass sample is simply-supported, the crack propagates into the top glass layer continuously until the collapse. During the experiment, rubber pads were placed between a sample and steel supports, which could provide some friction in the horizontal direction, which is not incorporated into the model. Unlike in the case of experimental testing, no unloading was assumed in the numerical model.

\section{CONCLUSIONS}

In this contribution, the tensile strength of the annealed and heat-strengthened glass in laminated glass plates was tested and a numerical model for glass fracture based on the phase-field technique was introduced. Based on the combined experimental and numerical analysis of three-layer laminated glass samples, the following conclusions can be done:

- This preliminary study proved that the introduced formulation was able to describe the behaviour of laminated glass under quasi-static bending well.

- The variability of tensile strength of glass strongly influences the response of laminated glass sample. Therefore, this variability should be incorporated into the model or discussed when the numerical results are interpreted.

The next step is to extend the proposed post-breakage model towards dynamics analysis of laminated glass under impact loading. For this study, a phase-field formulation based on tensile stress criterion was implemented to directly incorporate the results of the tensile strength evaluation into the model. For laminated glass plates, we are going to implement a model with the energy-based criterion from [13]. A disadvantage of this formulation is that softening caused by 
the damage evolution occurs even in the pre-critical range, and therefore, this elastic response is not linear. On the other hand, the main advantage of this model is its variational consistency.

\section{ACKNOWLEDGEMENTS}

This publication was supported by the Czech Science Foundation under project No. 19-15326S. We would also like to thank M. Eliášová, T. Hána, Z. Sokol, and V. Hrbek from CTU in Prague, the Centre of Excellence Telč, and the science centre AdMaS at TU Brno for performing the experimental measurements reported in this study.

\section{REFERENCES}

[1] Andreozzi, L., Bati, S.B., Fagone, M., Ranocchiai, G. \& Zulli, F., Dynamic torsion tests to characterize the thermo-viscoelastic properties of polymer interlayer for laminated glass. Construction and Building Materials, 65, pp. 1-13, 2014. https://doi.org/ 10.1016/j.conbuildmat.2014.04.003

[2] Janda, T., Zemanová, A., Zeman, J. \& Šejnoha, M., Finite element models for laminated glass units with viscoelastic interlayer for dynamic analysis. High Per-formance and Optimum Design of Structures and Materials II, WIT Transactions on The Built Environment, WIT Press, Vol. 166, pp. 245-254, 2016, ISSN 1746-4498.

[3] Schmidt, J., Janda, T. \& Šejnoha, M., Calibration of model for laminated glass polymer interlayer based on rheometer data. Experimental Stress Analysis 2017-Book of Full Texts, pp. 1-7, 2017.

[4] Melcher, J. \& Karmazínová, M., Experimentální verifikace procesu přetvárení a únosnosti plošných dílců s využitím metody zatěžování vakuováním, Z/B -Ověrená technologie. Faculty of Civil Engineering, Brno University of Technology, 2009. RIV/00216305:26110/09:PR24352.

[5] Vandebroek, M. \& Belis, J., Fracture strength of glass, engineering testing methods and estimation of characteristic values. COST Action TU0905 Mid-term Conference on Structural Glass, CRC Press, p. 223, 2013.

[6] Zemanová, A., Zeman, J. \& S`ejnoha, M., Comparison of viscoelastic finite element models for laminated glass beams. International Journal of Mechanical Sciences, 131-132, pp. 380-395, 2017. https://doi.org/10.1016/j.ijmecsci.2017.05.035

[7] Zemanová, A., Schmidt, J. \& Šejnoha, M., Evaluation of tensile strength of glass from combined experimental and numerical analysis of laminated glass. High Performance and Optimum Design of Structures and Materials III, WIT Transactions on The Built Environment, WIT Press, Vol. 175, pp. 29-39, 2018, ISBN 978-1-78466-289-9.

[8] Haldimann, M., Luible, A. \& Overend, M., Structural Use of Glass, volume 10 of Structural Engineering Documents. IABSE, Zurich, 2008.

[9] BS EN 16612, Glass in building-determination of the load resistance of glass panes by calculation and testing. Technical report, CEN/TC 129, 2013.

[10] Bourdin, B., Francfort, G.A. \& Marigo, J.J., Numerical experiments in revisited brittle fracture. Journal of the Mechanics and Physics of Solids, 48(4), pp. 797-826, 2000. https://doi.org/10.1016/s0022-5096(99)00028-9

[11] Borden, M.J., Verhoosel, C.V., Scott, M.A., Hughes, T.J. \& Landis, C.M., A phase-field description of dynamic brittle fracture. Computer Methods in Applied Mechanics and Engineering, 217-220, pp. 77-95, 2012. https://doi.org/10.1016/j.cma.2012.01.008

[12] Miehe, C., Schaenzel, L.M. \& Ulmer, H., Phase field modeling of fracture in multiphysics problems. part i. balance of crack surface and failure criteria for brittle crack 
propagation in thermo-elastic solids. Computer Methods in Applied Mechanics and Engineering, 294, pp. 449-485, 2015. https://doi.org/10.1016/j.cma.2014.11.016

[13] Kiendl, J., Ambati, M., De Lorenzis, L., Gomez, H. \& Reali, A., Phase-field description of brittle fracture in plates and shells. Computer Methods in Applied Mechanics and Engineering, 312, pp. 374-394, 2016. https://doi.org/10.1016/j.cma.2016.09.011

[14] Miehe, C., Hofacker, M. \& Welschinger, F., A phase field model for rate-independent crack propagation: Robust algorithmic implementation based on operator splits. Computer Methods in Applied Mechanics and Engineering, 199(45-48), pp. 2765-2778, 2010. https://doi.org/10.1016/j.cma.2010.04.011

[15] Zemanová, A., Zeman, J., Janda, T. \& Šejnoha, M., Layer-wise numerical modelfor laminated glass plates with viscoelastic interlayer. Structural Engineering and Mechanics, 65(4), pp. 369-380, 2018.

[16] Zemanová, A., Zeman, J., Janda, T., Schmidt, J. \& Šejnoha, M., On modal analysis of laminated glass: Usability of simplified methods and enhanced effective thickness. Composites Part B: Engineering, 151, pp. 92-105, 2018. https://doi.org/10.1016/j.compositesb.2018.05.032

[17] Duser, A.V., Jagota, A. \& Bennison, S.J., Analysis of glass/polyvinyl butyral laminates subjected to uniform pressure. Journal of Engineering Mechanics, 125(4), pp. 435-442, 1999. https://doi.org/10.1061/(asce)0733-9399(1999)125:4(435) 